Editorial

\title{
Special issue ESIAM19 theoretical and applied fracture mechanics
}

Additive manufacturing (AM) offers the potential to economically fabricate customized parts with complex geometries in a rapid designto-manufacture cycle. However, the basic understanding of the fracture behaviour of these materials must be substantially improved at all scale levels before the unique features of this rapidly developing technology can be used in critical load bearing applications. This ambitious target can be reached solely via adventurous interdisciplinary research. Our proposed project combines the strengths of materials science, technological processes, structural integrity assessment methodologies, and advanced design practices.

Innovative product design is believed to constitute the future revenue in the field of AM, where the ability to produce complex shaped parts facilitates light-weighting and other performance driven design opportunities. Whereas some work has been done on the fracture assessment and defect analysis of mostly metallic AM parts, the phenomena at place are extremely complex, not only driven by the intricate geometry but also by inherent process-property relationships. The inherent nature of the AM technology and the strong link between the evolving topology and its mechanical properties is by itself also a dilemma to be tackled. This unique feature makes it possible to tune a variety of properties beyond just the macroscopic appearance. Yet, it provides also unprecedented complexity in manufacturing that is difficult to be addressed in standards and recommendations to facilitate compliant design. Accordingly, the material and key mechanical properties of the fabricated parts are not necessarily generalizable, as they are strongly linked to the size and geometry of the part, besides the specific employed AM technology.

This limits both the theoretical understanding and structural applications and calls for thorough guidelines that are utilizable for practitioners, not necessarily involved in the particularities of the AM processes. To promote the utilization of this technology and to infuse product innovation in sectors such as automotive, biomedicine and aerospace, to facilitate shorter lead times and safer products at lower costs, the European Conference on the Structural Integrity of Additively Manufactured Materials, in short, ESIAM, was founded.

ESIAM 19 was the first conference of this series organized by ESIS TC15 on the topics of fatigue and fracture of AM materials. The objective has been to provide an updated state of the art on the development of effective criteria for the design of unprecedented high performing components for next generation automotive, aerospace and biomedical applications. The conference was successfully held in Trondheim from 9th to 11th of September 2019 with more than 150 participants from more than 20 countries. The present special issue contains invited papers from the conference that were subjected to the standard peer review process usually performed in Theoretical and Applied Fracture Mechanics. The main focus of the special issue is fracture mechanics, microstructure and defect analysis of additively manufactured materials under different loading scenarios. It is the first compendium of recent outcomes in the topic presented at the conference. A parallel special issue dealing with fatigue properties will be published in International Journal of Fatigue.

As guest editors, we want to express our acknowledgement to the authors, the reviewers and the editorial office staff that made this issue possible. We hope that the present issue provides a useful state of the art for engineers, academicians and industries involved in the challenges of producing high performant additively manufactured parts.

The next conference, ESIAM 21 will be held in Vienna Austria from 8th to 10th September 2021.

Sincerely yours

Filippo Berto Norwegian University of Science and Technology, Norway Jan Torgersen Norwegian University of Science and Technology, Norway

Matteo Benedetti University of Trento, Italy

Sara Bagherifard Politecnico di Milano, Italy

Chen Bo University of Leicester, United Kingdom

Guian Qian

${ }^{\mathrm{e}}$ Institute of Mechanics, Chinese Academy of Sciences, China E-mail address: filippo.berto@ntnu.no (F. Berto),

*Corresponding author. 